period 2, respectively. Surgery was the mainstay of treatment in both periods $(p=0.356)$. The adoption of minimally invasive surgery was consistent in the two study periods $(p=0.976)$. Before COVID-19 pandemic, 1,848 (72.8\%), 666 (26.3\%), and $25(0.9 \%)$ patients had minimally invasive, open and vaginal surgery, respectively. During the COVID-19 pandemic, 1,663 (72.8\%), $582(25.5 \%)$, and $41(1.7 \%)$ patients had minimally invasive, open, and vaginal surgery, respectively. Nodal assessment was omitted in $689(27.3 \%)$ and 484 (21.2\%) patients treated in period 1 and 2 , respectively $(p<0.001)$. While, the prevalence of patients undergoing sentinel node mapping (with or without backup lymphadenectomy) has increased during the COVID-19 pandemic (46.7\% in period 1 vs. $52.8 \%$ in period $2 ; \mathrm{p}<0.001)$. Overall, 1,280 (50.4\%) and 1,021 (44.7\%) patients had not adjuvant therapy in period 1 and 2 , respectively $(\mathrm{p}<0.001)$. Adjuvant therapy (in particular chemotherapy) use has increased during COVID-19 pandemic $(\mathrm{p}<0.001)$.

Conclusion* Our data suggest that the COVID-19 pandemic had a significant impact on the characteristics and patterns of care of EC patients. These findings highlight the need to implement healthcare services during the pandemic.

\section{CHARACTERIZATION OF ADVERSE REACTIONS IN PATIENTS WITH ADVANCED ENDOMETRIAL CANCER (AEC) RECEIVING LENVATINIB + PEMBROLIZUMAB (STUDY 309/KEYNOTE-775)}

N Colombo, ${ }^{3} \mathrm{D}$ Lorusso, ${ }^{4} \mathrm{AD}$ Santin, ${ }^{5} \mathrm{YM}$ Kim, ${ }^{6} \mathrm{AC}$ Herráez, ${ }^{7} \mathrm{~K}$ Yonemori, ${ }^{8} \mathrm{~K}$ Fujiwara, ${ }^{9} \mathrm{E}$ Colomba, ${ }^{10} \mathrm{DS}$ Miller, ${ }^{11} \mathrm{~S}$ Pignata, ${ }^{12} \mathrm{BJ}$ Monk, ${ }^{13} \mathrm{EM}$ Guerra, ${ }^{14} \mathrm{R}$ Kristeleit, ${ }^{15} \mathrm{M}$ Orlando,

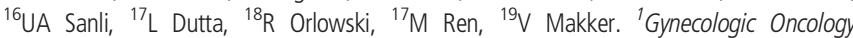
Program, University of Milan-Bicocca, European Institute of Oncology IRCCS, Milan, Italy; ${ }^{2}$ Gynecologic Oncology Program, University of Milan-Bicocca, European Institute of Oncology IRCCS, Milan, Italy; ${ }^{3}$ Division of Gynecologic Oncology, Fondazione Policlinico Universitario Agostino Gemelli IRCCS and Catholic University of Sacred Heart, Rome, Italy; ${ }^{4}$ Department of Obstetrics, Gynecology and Reproductive Sciences, Yale University School of Medicine, New Haven, USA; ${ }^{5}$ Department of Obstetrics and Gynecology, Asan Medical Center, University of UIsan, Seoul, Korea, Rep. of South; ${ }^{6}$ Department of Medical Oncology, San Carlos University Teaching Hospital, Madrid, Spain; ' Department of Breast and Medical Oncology, National Cancer Center Hospital: Kokuritsu Gan Kenkyu Center Chuo Byoin, Tokyo, Japan; ${ }^{8}$ Department of Gynecologic Oncology, Saitama Medical University International Medical Center, Hidaka, Japan; ${ }^{9}$ Department of Cancer Medicine, Gustave Roussy Cancerology Institute, Villejuif, GINECO group, France; ${ }^{10}$ Division of Gynecologic Oncology, University of Texas Southwestern Medical Center, Dallas, USA; ${ }^{11}$ Department of Urology and Gynecology, Istituto Nazionale Tumori IRCCS-Fondazione G. Pascale, Naples, Italy; ${ }^{12}$ Arizona Oncology (US Oncology Network), University of Arizona, Creighton University, Phoenix, USA; ${ }^{13}$ Senvicio de Oncología Médica, Hospital Universitario Ramón y Cajal, Madrid, Spain; ${ }^{14}$ Department of Oncology, Guy's and St Thomas' NHS Foundation Trust, London, UK; ${ }^{15}$ Oncologia Clinica, Instituto Alexander Fleming, Buenos Aires, Argentina; ${ }^{16}$ Department of Medical Oncology, Ege University, Izmir, Turkey; ${ }^{17}$ Clinical Research, Eisai Inc., Wooddliff Lake, USA; ${ }^{18}$ Late Stage Clinical Development, Merck and Co., Inc., Kenilworth, USA; ${ }^{19}$ Department of Medicine, Memorial Sloan Kettering Cancer Center; Weill Cornell Medical Center, New York, USA

\subsection{6/ijgc-2021-ESGO.120}

Introduction/Background* In Study 309/KEYNOTE-775, lenvatinib + pembrolizumab showed significant and clinically meaningful improvements in OS, PFS, and ORR versus treatment of physician's choice (TPC) in aEC patients following prior platinum-based therapy. Safety considerations are also important in EC. Herein, we characterize common adverse reactions (ARs) in patients with aEC in Study 309/KEYNOTE-775 and their respective management strategies. Additionally, the clinician's role in proactively managing ARs will be highlighted.
Methodology In Study 309/KEYNOTE-775, patients were randomized to lenvatinib $20 \mathrm{mg}$ QD $\mathrm{PO}+$ pembrolizumab $200 \mathrm{mg}$ IV Q3W $(\mathrm{n}=411)$ or TPC $(\mathrm{n}=416$; doxorubicin 60 $\mathrm{mg} / \mathrm{m}^{2}$ IV Q3W or paclitaxel $80 \mathrm{mg} / \mathrm{m}^{2}$ IV QW, 3 weeks on/1 week off). Herein, characterization of key ARs is based on incidence and known association with lenvatinib+pembrolizuumab, and interventions for ARs in aEC patients. Key ARs are grouped by preferred terms per FDA definitions for ARs in patients with endometrial carcinoma from the US prescribing information; ARs include hypertension, musculoskeletal pain, fatigue, nausea, diarrhea, decreased appetite, stomatitis, vomiting, hypothyroidism, palmar-plantar erythrodysesthesia (PPES), and decreased weight.

Result(s)* Median times (weeks) to first onset of key ARs [any grade] were: hypertension (2.1), fatigue (2.3), musculoskeletal pain (3.2), nausea (4.7), decreased appetite (4.9), stomatitis (4.9), vomiting (7.6), diarrhea (7.9), hypothyroidism (8.9), PPES (9.6), and decreased weight (10.7). Among ARs described, those that led to withdrawal of lenvatinib included decreased appetite (2\%), fatigue (2\%), hypertension (2\%), diarrhea (1\%), musculoskeletal pain $(1 \%)$, vomiting $(1 \%)$, and decreased weight (1\%); only decreased appetite (1\%) and diarrhea $(1 \%)$ led to withdrawal of pembrolizumab. Hypertension most frequently led to lenvatinib dose reduction (18\%); diarrhea and hypertension most frequently led to dose interruption of lenvatinib (11\% each) as last action taken with lenvatinib. Diarrhea most frequently led to pembrolizumab interruption (8\%). Change in sum of target lesion diameters over time, exposure-adjusted ARs, and AR management strategies will be reported.

Conclusion* In general, ARs due to lenvatinib+pembrolizumab were as expected and often occurred within 3 months of treatment initiation. As will be presented, clinicians play a critical role in prompt identification and AR-directed management of patients with aEC; such management may potentially reduce treatment interruption(s) and/or lenvatinib dose reduction.

\section{INCREASED SURVIVAL IN NON-ENDOMETRIOID ENDOMETRIAL CANCER AFTER INTRODUCTION OF SWEDISH NATIONAL GUIDELINES}

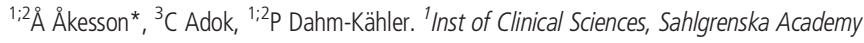
at the University of Gothenburg, Obstetrics and gynecology, Göteborg, Sweden: ${ }^{2}$ Sahlgrenska University Hospital, Gynecology, Göteborg, Sweden; ${ }^{3}$ Regionalt cancercentrum väst, Göteborg, Sweden

\subsection{6/ijgc-2021-ESG0.121}

Introduction/Background* The first Swedish national guidelines for endometrial cancer (NGEC) recommended adequate staging with pelvic and paraaortic lymphadenectomy for patients with high-risk disease, including non-endometrioid endometrial cancer (EC). The recommended adjuvant oncological treatment protocol was chemotherapy to all non-endometrioid EC and radiotherapy only for those with stage IIIC. Before the NGEC, the stipulated surgery was solely hysterectomy and bilateral salpingectomy followed by adjuvant chemo-and radiotherapy to all non-endometrioid ECs. The aim of this study was to investigate the outcome in survival and recurrence of this shift in treatment strategy. 